\title{
Interpretation of salt bodies using semi-supervised deep convolutional neural network and graph cuts for segmentation
}

\author{
Allan Segovia Spadini, Alura
}

\begin{abstract}
Copyright 2021, SBGf - Sociedade Brasileira de Geofísica
This paper was prepared for presentation during the $17^{\text {th }}$ International Congress of the Brazilian Geophysical Society held in Rio de Janeiro, Brazil, 16-19 August 2021.

Contents of this paper were reviewed by the Technical Committee of the $17^{\text {th }}$ International Congress of the Brazilian Geophysical Society and do not necessarily represent any position of the SBGf, its officers, or members. Electronic reproduction or storage of any part of this paper for commercial purposes without the written consent of the Brazilian Geophysical Society is prohibited.
\end{abstract}

\begin{abstract}
In this study, the segmentation of salt structures in seismic images was analyzed through the combination of two techniques for image segmentation. The first technique was the graph cut. This technique is an interesting technique for the segmentation of salt structures. Even in situations with little user input, it was possible to cut the geometric structures of the images accurately. The second technique was the application of convolutional layers for semantic segmentation. Convolutional networks were applied in a semi-supervised manner. The output of the graph cutting application was used to direct the simplification of the image provided by the convolutional layers. The individual application of each technique is not perfect and the combination of the two together outperforms the results.
\end{abstract}

\section{Introduction}

Image segmentation involves identifying the nature of each pixel in an image. The purpose of image segmentation is to simplify and/or change the representation of the image so that interpretation becomes easier. The interpretation of faults and fractures and also of salt bodies in seismic data can benefit from this type of technique. However, the segmentation and classification of these characteristics in seismic images still require the presence of specialized professionals such as geologists and geophysicists even with the extraction of information such as seismic attributes from the data.

The expansion of machine learning techniques, more specifically deep learning, has made it possible to build models capable of classifying structures in seismic images. The disadvantage of applying deep learning classification algorithms is that the data used for training the algorithms need to be manually labeled. Also, the presence of public and annotated seismic data is scarce. An alternative is the development of unsupervised techniques to solve this problem.

The automatic interpretation of salt bodies through deep learning techniques has been gaining interest in recent years. Waldeland and Solberg (2017) applied convolutional networks for the segmentation of salt bodies and Waldeland et al. (2018) showed in detail how deep learning can be used to interpret salt bodies through supervised learning with convolutional neural networks. Shi et al. (2019) formulated the salt segmentation problem as a 3D image segmentation through an encoder-decoder architecture with convolutional neural networks.

In contrast, unsupervised algorithms have been applied in different areas for a long time. Coléou et al. (2003) reviewed the application of unsupervised methods to the classification of seismic facies. As an alternative to the application of supervised algorithms, Babakhin et al. (2019) proposed the semi-supervised segmentation of salt bodies. They used a set of convolutional neural networks and created pseudo-labels to enable the semantic segmentation of salt. Kim et al. (2020) applied convolutional networks to general-purpose images in an unsupervised algorithm. The criteria adopted for this segmentation were that pixels that received the same label should have similar characteristics and be spatially continuous. In addition, they adopted the criterion that the number of unique labels should be large.

Convolutional neural networks can extract characteristics relevant to image classification. But in an unsupervised problem there is no clue of what features of the image should be emphasized. The focus of this work was the application of unsupervised algorithms for the segmentation of salt bodies. This problem has unique characteristics. For this reason, in this paper, two different techniques were combined to improve the segmentation of the image.

\section{Method}

\section{Graph Cuts}

One of the techniques studied was the graph cut technique. The algorithm used was proposed in Rother et al. (2004). The operation of this type of algorithm can be illustrated in Figure 1. In this figure, each pixel of the image is transformed into a node of a graph. Each node of this graph is connected to a T terminal that represents the background and to an $S$ terminal that represents an object. The connection between the nodes and the terminals receives a value of the probability of belonging to the background of the object. Then the pixels are linked to their neighboring pixels. The connection between neighboring pixels also receives weights that promote the connection of similar pixels. After the construction of the graph, this graph is partioned. An example of the cutting algorithm used is the minimum cut used in Boykov; Jolly (2001). 

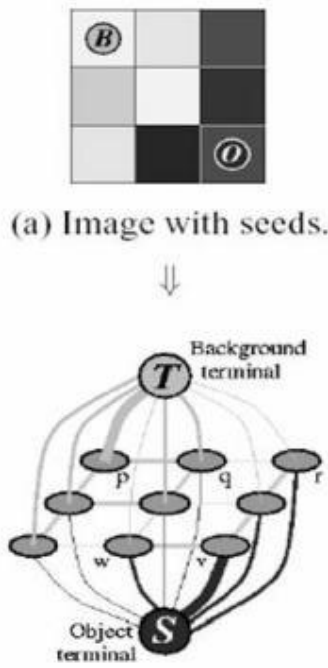

(b) Graph. (a) Image with seeds.

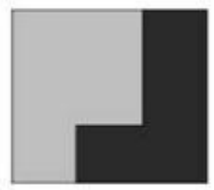

(d) Segmentation results.
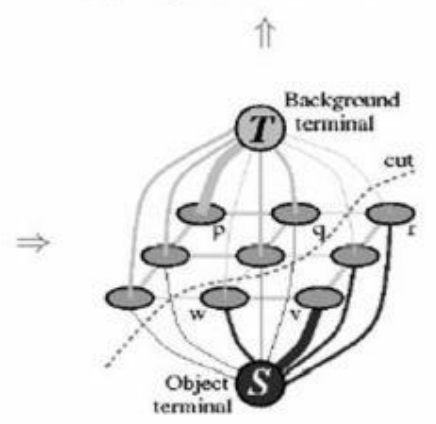

(c) Cut.
Figure 1 - Illustration of the operation of the Grabcut algorithm. Source: Marsh et al. ()

\section{Unsupervised convolutional scheme}

The convolutional neural network prosed by Kim et al. (2020) was used as a basis for this study. Figure 2 illustrates the network architecture. First, the same label is added to pixels with similar characteristics. This is done through a linear classifier that addresses different classes to pixels. The algorithm starts with 100 classes added to the image's pixels.

With each iteration of the algorithm, the convolutional layers act as approximators of the characteristics of the pixels present. This causes the number of classes to decrease. To control the evolution of the algorithm, a cost function is used. This cost function tries to control two different characteristics. The first is the similarity 0 the image characteristics and the second is the spatial continuity of the image. We can write the $L$ cost function like this:

$$
\mathrm{L}=\mathrm{L}_{\text {sim }}+\mu \mathrm{L}_{\text {con }} \text {. }
$$

In equation (1) $L_{\text {sim }}$ is the cost of similarity, $L_{\text {con }}$ the continuity cost and $\mu$ is the weight that relates the two cost functions. For the cost function that takes into account the similarity, the cross-entropy function was used. For the function that considers continuity, an L1 loss was used.

\section{Joint application}

The application of convolutional networks tends to bring together the different classes in the image. This can be desired or not depending on the structures we want to observe. Thus, the output of the application of the Grabcut algorithm was used as the input RGB image. This will provide larger segments in the images. Keeping the segmented image by Grabcut in the first iterations means that the convolutional layers do not make major changes in these regions of the image.

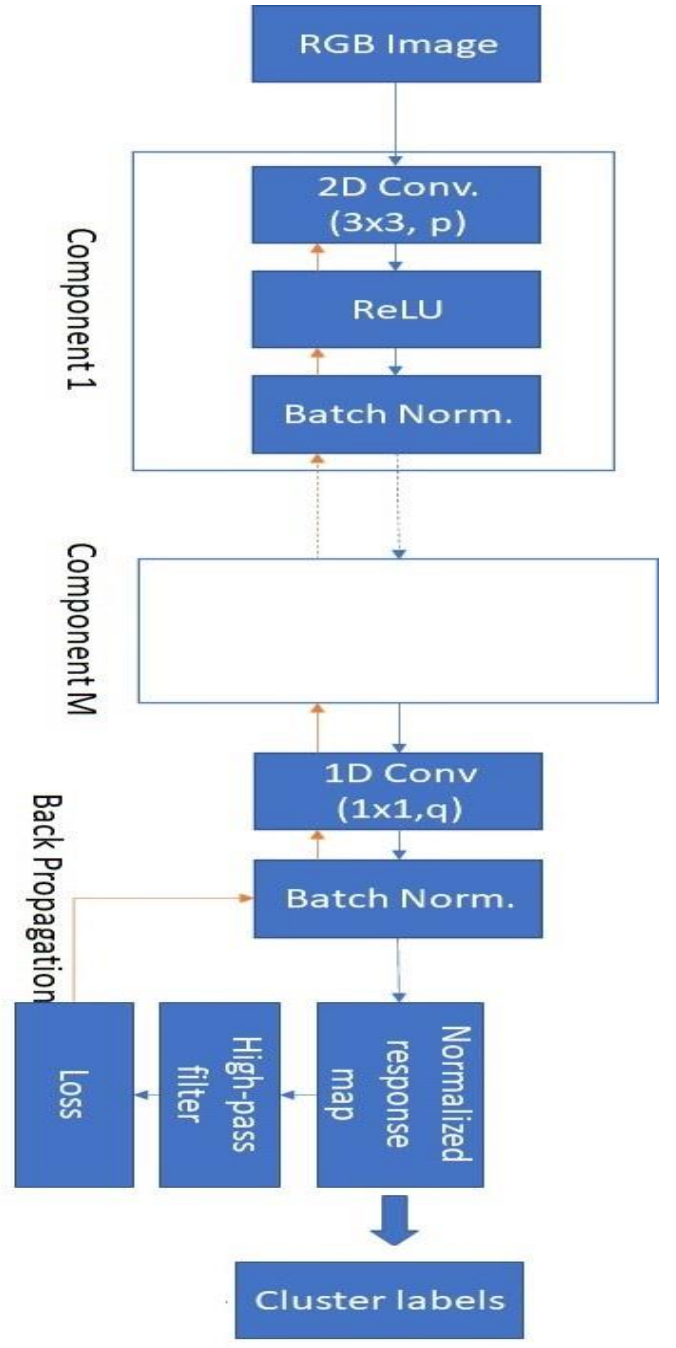

Figure 2 - Architecture of the set of convolutional layers used for semantic segmentation.

\section{Results}

To test the application of the methodology, a section of the SEG Advanced Modeling (SEAM) Phase I synthetic data migrated image detailed in Fehler and Keliher (2011) was used. The input image used for the Grabcut algorithm is shown in Figure 3. The central structure in the image is the object of interest in the image.

For the application of graph cut, a rectangle containing the object of interest was passed. The entire image external to the rectangle is considered background. The adoption of a rectangle that leads to a segmentation of the salt structure was made by trial and error. This is the only part that requires manual user input.

The application of this type of algorithm also involves the adoption of scribbles. Those scribbles define regions where the background and the object of interest are. Thus, this returns more precise segmentation results. However, the goal is to get as close as possible to an unsupervised algorithm. Because of that, the only input was the rectangle. Defining an input rectangle that returned a good 
segmentation of the salt structure was difficult and required several attempts. The final rectangle adopted starts at the coordinates $(0,0)$ and has a length equal to 500 pixels and a height equal to 700 pixels.

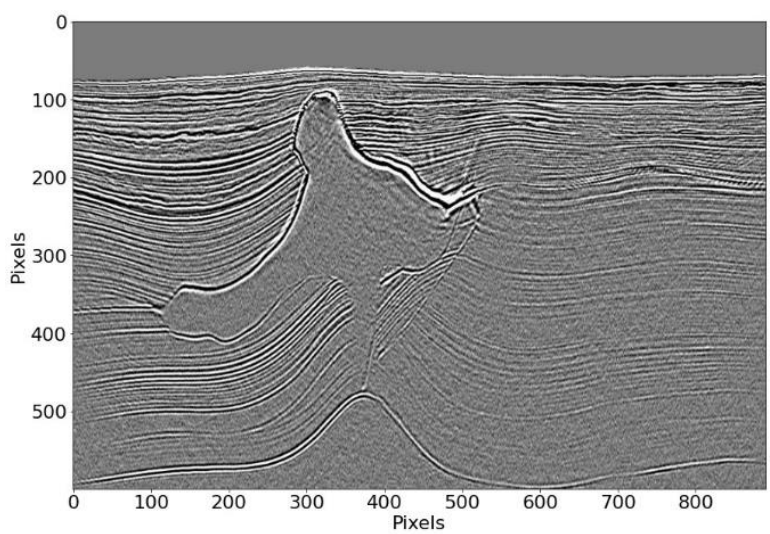

Figure 3 - 2D Cross-section of SEAM synthetic data used as input.

After applying the algorithm, the image in Figure 4 was obtained. This image is the difference between the output of the Grabcut algorithm and the original image shown in Figure 3 . The image in figure 4 is used as an input to the segmentation algorithm using convolutional layers.

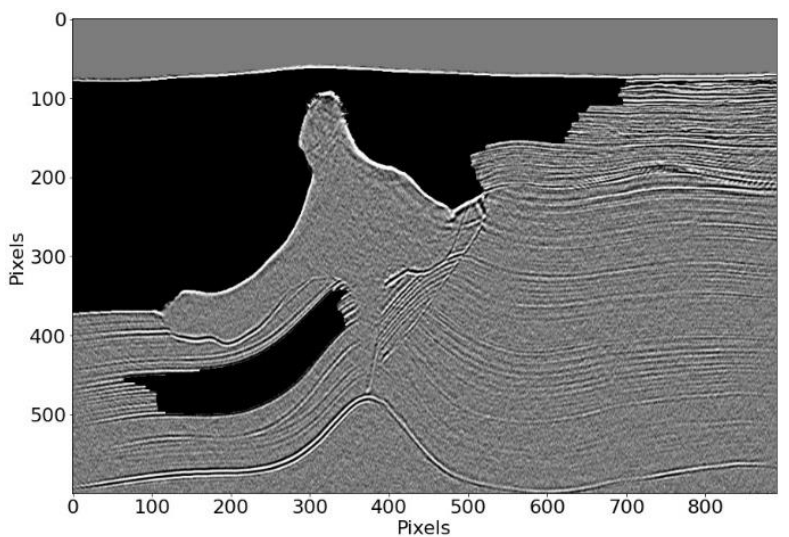

Figure 4 - Difference between the original input and the Grabcut output.

The reason why the difference between the original entrance and the exit o Grabcut was chosen is that the image in Figure 4 has a black region surrounding the salt structure. When this image is used as input in the convolutional algorithm, the black region defines a single class around the image.

The convolutional algorithm tends to simplify the image. Defining a region around the structure as a single class prevents this region from being simplified in the first iterations of the algorithm. After 5 iterations of the algorithm, the image in Figure 4 has been replaced by the image in Figure 3. This way, the layers around the salt structure will not be so simple even in the course of more iterations of the convolutional algorithm.

As can be seen in Figure 5, the structures that belonged to the image in both Figure 3 and Figure 4 were simplified. This is even more evident when we look at Figure 6 where the algorithm ran for more iterations. In the upper right part of Figures 5 and 6 , we have an additional simplification of the image. This simplification is just because these structures ate also present in Figure 4.

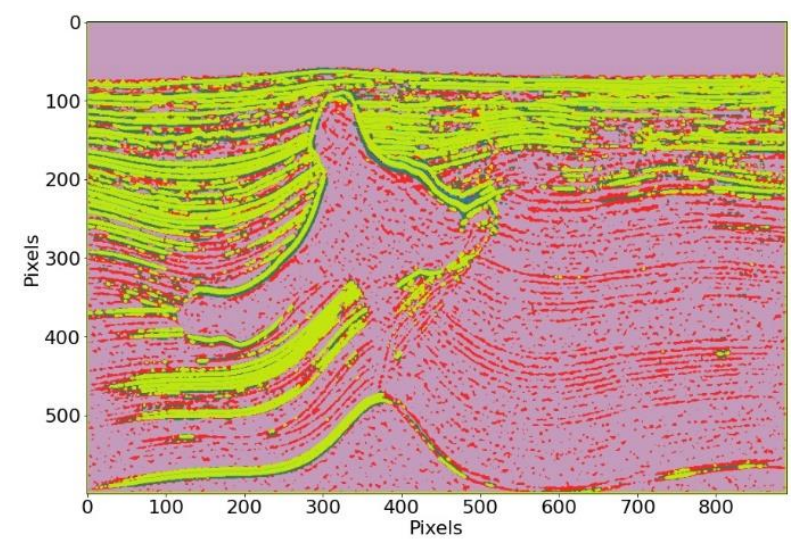

Figure 5 - Output from the convolutional layer algorithm after 100 iterations.

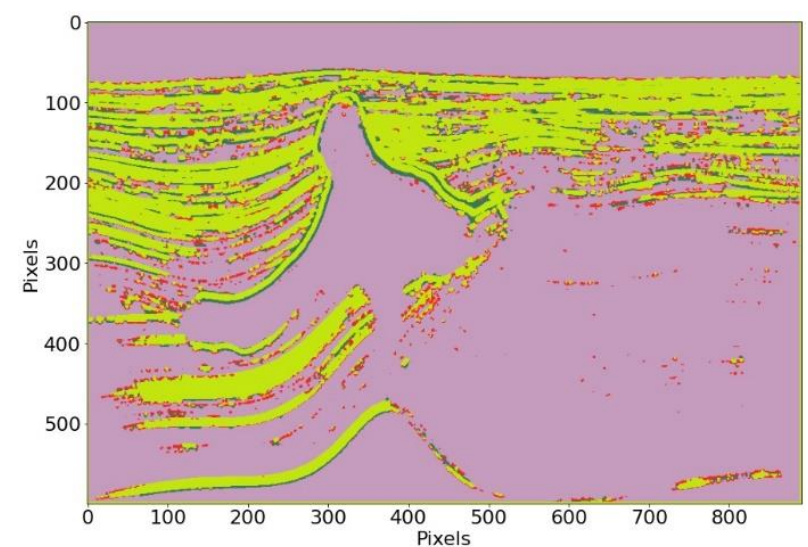

Figure 6 - Output from the convolutional layer algorithm after 200 iterations.

To give a better view of the evolution of the convolutional layers algorithm, the cost function and the number of classes in the image also show during the iterations of the algorithm. In figures 7 and 8 , it is possible to observe that the number of classes changes after the fifth iteration of the algorithm. However, this does not have a measurable impact on the cost function. 


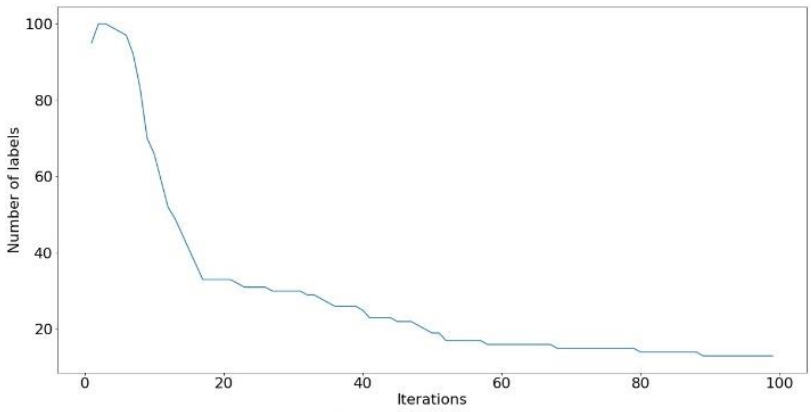

Figure 7 - Number of labels in the image through iterations of the convolutional layers algorithm.

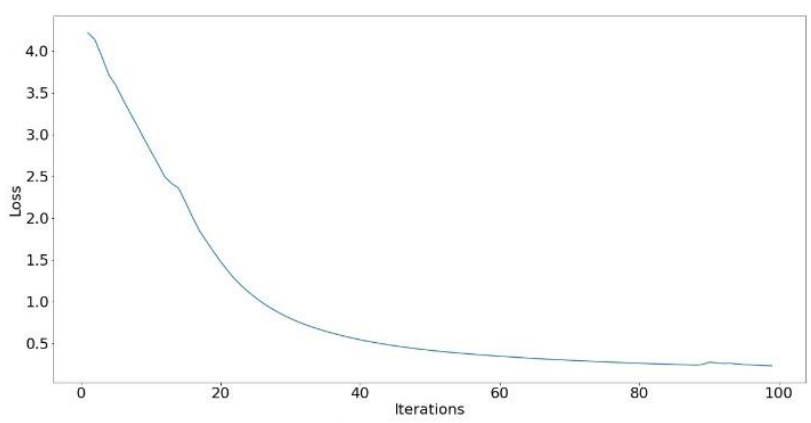

Figure 8: Cost function of the convolutional layers algorithm through iterations.

\section{Conclusions}

The application of algorithms that try to extract image information without training with several examples is difficult. Both the Grabcut algorithm and the convolutional layer algorithm show promising characteristics in image segmentation. Likewise, its joint application is interesting since it makes it possible to focus on certain structures of interest without losing certain characteristics of the data such as sequences of sedimentary layers. However, segmentation still has room for improvement through obtaining measures that allow a better definition of the target of interest. The application of Grabcut is the most promising because it can cut the geometric structures of the image with precision when we can define well what is the background of the image.

The convolutional layer algorithm is more challenging. Convolutional neural networks can extract interesting features from images and are very efficient in supervised algorithms. However, it is still necessary to establish a criterion that works well with the application of convolutional filters and provides good identification of structures of a certain type in an image.

\section{References}

BABAKHIN, Y.; SANAKOYEU, A.; KITAMURA, H. SemiSupervised Segmentation of Salt Bodies in Seismic Images using an Ensemble of Convolutional Neural Networks. , 2019. Disponível em: <http://arxiv.org/abs/1904.04445>. .

BOYKOV, Y. Y.; JOLLY, M.-P. Interactive graph cuts for optimal boundary \&amp; region segmentation of objects in $\mathrm{N}-\mathrm{D}$ images. Proceedings Eighth IEEE International Conference on Computer Vision. ICCV 2001. Anais... . v. 1, p.105-112, 2001. IEEE Comput. Soc. Disponível em: $<$ http://ieeexplore.ieee.org/document/937505/>. .

COLÉOU, T.; POUPON, M.; AZBEL, K. Unsupervised seismic facies classification: A review and comparison of techniques and implementation. The Leading Edge, v. 22, n. 10, p. 942-953, 2003. Disponível em: <http://library.seg.org/doi/10.1190/1.1623635>. .

FEHLER, M.; KELIHER, P. J. SEAM Phase 1: Challenges of Subsalt Imaging in Tertiary Basins, with Emphasis on Deepwater Gulf of Mexico. Society of Exploration Geophysicists, 2011.

KIM, W.; KANEZAKI, A.; TANAKA, M. Unsupervised Learning of Image Segmentation Based on Differentiable Feature Clustering. IEEE Transactions on Image Processing, v. 29, p. 8055-8068, 2020. Disponível em: $<$ https://ieeexplore.ieee.org/document/9151332/>. .

MARSH, M.; BANGAY, S.; LOBB, A. Grabcut. Disponível em: <https://www.cs.ru.ac.za/research/g02m1682/>. Acesso em: 1/1/2021.

ROTHER, C.; KOLMOGOROV, V.; BLAKE, A. "GrabCut": interactive foreground extraction using iterated graph cuts. ACM SIGGRAPH 2004 Papers on - SIGGRAPH '04. Anais... . p.309, 2004. New York, New York, USA: ACM Press. Disponível em: $<$ http://portal.acm.org/citation.cfm?doid=1186562.101572 $0>$. .

SHI, Y.; WU, X.; FOMEL, S. SaltSeg: Automatic 3D salt segmentation using a deep convolutional neural network. Interpretation, v. 7, n. 3, p. SE113-SE122, 2019. Disponível em: <https://library.seg.org/doi/10.1190/INT2018-0235.1>. .

WALDELAND, A. U.; JENSEN, A. C.; GELIUS, L.-J.; SOLBERG, A. H. S. Convolutional neural networks for automated seismic interpretation. The Leading Edge, v. 37, n. 7, p. 529-537, 2018. Disponível em: <https://library.seg.org/doi/10.1190/tle37070529.1>. .

WALDELAND, A. U.; SOLBERG, A. H. S. S. Salt Classification Using Deep Learning. , 2017. Disponível em:

$<$ http://www.earthdoc.org/publication/publicationdetails/?p ublication $=88635>$. . 